\title{
PERFUSION FIXATION WITH GLUTARALDEHYDE AND POST-FIXATION WITH OSMIUM TETROXIDE FOR ELECTRON MICROSCOPY
}

\author{
A. VAN HARREVELD AND F. I. KHAT'TAB* \\ The Kerckhoff Laboratories of the Biological Sciences, California Institute of Technology, \\ Pasadena, California, U.S.A.
}

\section{S U M MARY}

The conductivity of cerebral cortex drops during perfusion with glutaraldehyde in 5 min to about $60 \%$ of the original value, to remain unchanged during the subsequent $10-15 \mathrm{~min}$ of perfusion. Circulatory arrest causes a similar drop in the tissue conductivity. Perfusion of asphyxiated tissue with glutaraldehyde does not produce additional major changes in the conductivity. Perfusion of the cortex with an osmium tetroxide solution causes an initial drop in conductivity. However, after about $3 \mathrm{~min}$ this trend is reversed and the conductivity increases again to close to the pre-perfusion value. Perfusion of asphyxiated cortex with $\mathrm{OsO}_{4}$ causes a marked increase of the conductivity. So does perfusion with an $\mathrm{OsO}_{4}$ solution of tissue previously treated with glutaraldehyde.

One interpretation of these impedance changes is that glutaraldehyde perfusion causes, like asphyxiation, a transport of extracellular material into the intracellular compartment and that during $\mathrm{OsO}_{4}$ perfusion an extracellular space is again created. This possibility is supported by electron micrographs made of this material. Cerebral cortex perfused with glutaraldehyde and post-fixed with $\mathrm{OsO}_{4}$ shows electron-transparent dendritic elements and to a lesser extent pre-synaptic terminals, which seem to be swollen. When the cortex is flooded with a salt solution during glutaraldehyde perfusion the dendrites exhibit ballooning in the surface layer of the cortex, suggesting that the fluid on the cortex participates in the swelling. The tissue elements in the glutaraldehyde-perfused and $\mathrm{OsO}_{4}$ post-fixed cortex are separated by narrow extracellular spaces. The latter may have been produced by the $\mathrm{O}_{8} \mathrm{O}_{4}$ perfusion as is suggested by a comparison of micrographs prepared by freeze substitution (which tends to preserve the water distribution) of glutaraldehyde-perfused but not post-fixed cortex with micrographs of cortex treated with $\mathrm{OsO}_{4}$ after the glutaraldehyde perfusion. In accordance with the conductivity changes, the former micrographs showed very little extracellular space, and in many places tight junctions, whereas the latter showed clefts between the tissue elements.

\section{INTRODUCTION}

The lability of the water distribution in central nervous tissue is demonstrated by the marked differences of electron micrographs (EMs) of cerebellar and cerebral cortex rapidly frozen and subjected to freeze substitution shortly after circulatory arrest as compared with tissue frozen after a period of asphyxiation. Such micrographs suggested that during a short $(6-8 \mathrm{~min})$ period of circulatory arrest a transport occurs of extracellular material into certain cellular elements, such as dendrites in the cerebral and cerebellar cortex and fibres of Bergmann in the latter tissue, which appeared electron-transparent and enlarged in electron micrographs of asphyxiated

- Present address: Zoology Dept., Ain Shams University, Cairo, Egypt (UAR). 
tissue as compared with the same elements in non-asphyxiated material. Furthermore, there was present an appreciable extracellular space in EMs of non-asphyxiated tissue which was greatly reduced after asphyxiation (Van Harreveld, Crowell \& Malhotra, 1965; Van Harreveld \& Malhotra, 1967). The postulate that during asphyxiation extracellular material is transported into the intracellular compartment was supported by the observation of a movement of chloride (probably accompanied by sodium) from a presumably extracellular space into dendrites and fibres of Bergmann (Van Harreveld \& Schadé, 1959; Van Harreveld, 196I), and by a swelling of the same elements (Van Harreveld, 1957, 1961). Similar movements of chloride and water into apical dendrites were observed in the cerebral cortex during spreading depression (Van Harreveld, 1958; Van Harreveld \& Schadé, 1959). Also a loss of extracellular space and a swelling of dendritic elements was found in electron micrographs of cerebral cortex frozen during spreading depression (Van Harreveld \& Khattab, 1967).

Concomitant with the transport of extracellular material into cellular elements during asphyxiation and spreading depression the electrical impedance of the tissue increases markedly (Leão \& Ferreira, I 953; Freygang \& Landau, 1955; Van Harreveld \& Ochs, 1956, 1957; Ranck, 1964). This impedance increase can be explained by the loss of extracellular electrolytes which carry most of the measuring current used in impedance determinations. The intracellular electrolytes are surrounded by cell membranes which impede ion movements and in this way limit their participation in the current transport. A shift from an extra- to an intracellular position can therefore be expected to enhance the tissue impedance (Van Harreveld \& Ochs, 1956; Van Harreveld, ig66).

If a mild procedure such as a few minutes of asphyxiation or the more physiological changes involved in spreading depression are able to cause a shift of extracellular material into the intracellular compartment, then it would not be surprising if the strong stimulus of perfusing the tissue with the fixatives for electron microscopy affects the water and electrolyte distribution in central nervous tissue. Such an effect would explain the paucity of the extracellular space in electron micrographs of central nervous tissue fixed in this way (see, for example, Karlsson \& Schultz, I965), which contrasts with the relative abundance of extracellular material observed in freezesubstituted tissue of non-asphyxiated cerebellar and cerebral cortex (Van Harreveld et al. 1965; Van Harreveld \& Malhotra, 1967). A marked increase of the cortical impedance during perfusion of the tissue with formalin (Van Harreveld, I966) supports the concept that a loss of extracellular material occurs during fixation. In an investigation of perfusion fixation with a number of fixatives combined with a variety of buffers and other additives Nevis \& Collins (1967) observed in general an initial increase of the tissue resistance which was maintained with some combinations of fixatives and buffers, and reversed with others. In the present experiments the effects of perfusion fixation with glutaraldehyde and osmium tetroxide on the tissue impedance were investigated. EMs of the fixed tissues were compared with the results obtained by freeze substitution of cerebral and cerebellar cortex. 
METHODS

All experiments were carried out on albino mice narcotized with urethane. To perfuse the brain the abdomen was opened widely, the abdominal aorta was dissected and a ligature placed around this vessel between the superior mesenteric and renal arteries. The aorta was clamped cranial of this region, a metal cannula was inserted into the vessel and tied in place. The cannula was connected with the perfusion apparatus consisting of two containers, one filled with Ringer's solution, the other with the fixative. The cannula itself was filled with a heparin solution to prevent the formation of a blood clot. The brain case was opened widely over one hemisphere using a highspeed drill, after which the dura was removed with jeweller forceps (Van Harreveld \& Malhotra, 1967). The wide opening of the cranium prevents a pressure build-up in the brain case due to possible swelling of the tissue, which could impair the perfusion. In most experiments the unexposed cortex was examined but in some the exposed surface was used. To start perfusion, pressure (about $\mathrm{I} 3 \mathrm{~cm}$ mercury) was applied to solutions in the perfusion apparatus; then the clamp on the aorta was removed. The thorax was opened and the right heart was incised to allow escape of the perfusion solution. The preparation was perfused for about ro sec with Ringer's solution, then with the fixative.

The tissue was perfused with glutaraldehyde $5 \%$ in a $\mathrm{I} / \mathrm{I} 5 \mathrm{M}$ phosphate buffer $(\mathrm{pH} 7 \cdot 4)$. Sodium chloride $(4 \%)$ was added to give the perfusion solution a similar electrical conductivity to that of the plasma $\left(66 \Omega \mathrm{cm}\right.$ at $\left.38^{\circ} \mathrm{C}\right)$. The osmo concentration of the fixative was about $800 \mathrm{~m}$-osmols. Perfusion was maintained for $15^{-20} \mathrm{~min}$. This caused sometimes a mild swelling of the tissue as shown by a slight protrusion of the exposed hemisphere. In other experiments apparently no changes or a slight decrease in tissue volume occurred. Blocks were cut of the unexposed (and in some instances from the exposed) cerebral cortex and of the cerebellar vermis which were post-fixed with osmium tetroxide $(2 \%)$ in the phosphate buffer. In a few experiments the brain was perfused with a $\mathrm{I} \% \mathrm{OsO}_{4}$ solution in the phosphate buffer with $4 \% \mathrm{NaCl}$ added to make the conductivity of the solution similar to that of the plasma. The fixed blocks were dehydrated in alcohol and embedded in Maraglas (Freeman \& Spurlock, 1962). Thick $(\mathrm{I}-\mu)$ sections were cut on an LKB Ultrotome and, after staining with methylene blue and azure II (Richardson, Jarett \& Finke, 1960), were studied with the light microscope. Thin sections were studied, after staining with lead citrate (Reynolds, 1963), with a Philips EM 200 electron microscope.

In some experiments perfusion fixation was combined with freezing of the tissue and replacement of the ice with acetone at low temperature $\left(-85^{\circ} \mathrm{C}\right)$. The procedure in which the tissue surface is made to contact a silver surface kept at about $-207^{\circ} \mathrm{C}$ has been described previously (Van Harreveld \& Crowell, 1964; Van Harreveld et al. 1965).

Tissue impedance was measured with an alternating current bridge at $1000 \mathrm{c} / \mathrm{s}$ (Van Harreveld \& Ochs, 1956). Small silver-silver chloride electrodes placed on the cortical surface were used. The measurements were carried out in a room kept at 
$38^{\circ} \mathrm{C}$ with the perfusion solutions warmed to the same temperature, to prevent conductivity changes due to changes in temperature of the tissue.

A total of 95 mice were used in this investigation.

\section{RESULTS}

\section{Impedance measurements}

The cortical impedance was measured for a few minutes before perfusion was started and frequent measurements were taken during the perfusion. In Fig. I A the reciprocal of the impedance, the conductivity, which is more directly related to the amount of extracellular electrolytes (Van Harreveld, I966), is plotted against time during glutaraldehyde perfusion. The first effect is a slight and transient increase of

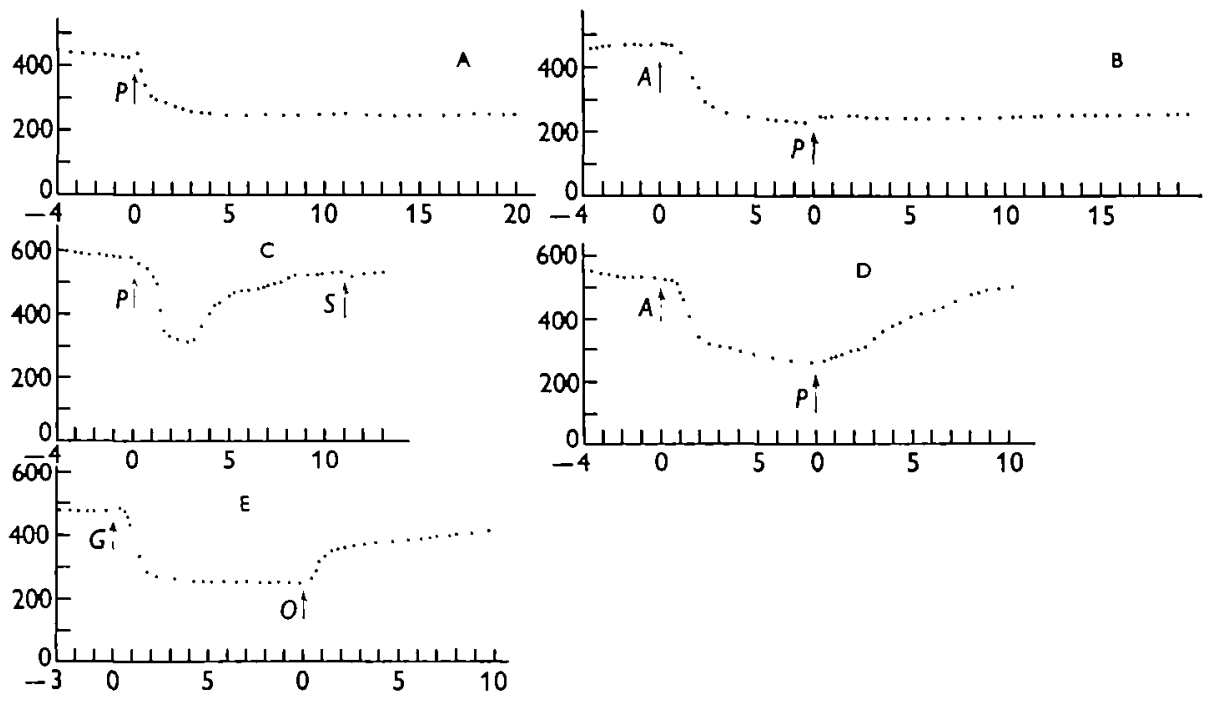

Fig. I. Five graphs ( $A$ to $E$ ) in which the conductivity is plotted on the ordinate in mhos $\times 10^{8}$, and time in min is plotted on the abscissa. A, At the arrow $(P)$ the head is perfused with Ringer's solution for ro sec immediately after circulatory arrest, then with glutaraldehyde. B, At the first arrow $(A)$ the circulation is arrested; at the second arrow $(P)$ the head is perfused with glutaraldehyde after having been perfused with Ringer's solution for ro sec. $\mathrm{C}$, At the first arrow $(P)$ the head is perfused for ro sec with Ringer's solution, then with osmium tetroxide; at the second arrow $(S)$ the perfusion is stopped. D, At the first arrow $(A)$ the circulation is arrested; at the second arrow $(P)$ the head is perfused with Ringer's solution for 10 sec followed by $\mathrm{OsO}_{4}$ perfusion. E, At the first arrow $(G)$ the head is perfused first with Ringer's solution for Io sec, then with glutaraldehyde; at the second arrow $(O)$ the head is perfused with $\mathrm{OsO}_{4}$.

the conductivity. This may be due to the replacement of the blood in the vessels with the perfusion fluid, which has a similar conductivity to plasma but a higher conductivity than blood due to the absence of the erythrocytes. However, very soon (within a fraction of a minute) the conductivity starts to drop, reaching in $5 \mathrm{~min}$ a value of $58 \%$ of the pre-perfusion conductivity. During the rest of the perfusion period ( $20 \mathrm{~min})$ 
this value is approximately maintained. The perfusion not only brings the tissue in contact with the fixative, it also interferes with its oxygen supply, which is known to cause a marked conductivity drop (Van Harreveld \& Ochs, 1956). The latter effect has a latency of $\mathrm{I}$ to $2 \mathrm{~min}$ in the mouse (Van Harreveld \& Malhotra, I967). The early start of the conductivity drop during the perfusion with glutaraldehyde suggests therefore that the impedance change was not caused by oxygen lack, but by the action of the fixative. Nevis \& Collins ( 1967 ) reported a similar conductivity loss during glutaraldehyde perfusion.

Table I. Conductivity changes (as a percentage of the value measured in the normal, oxygenated cortex) after perfusion with glutaraldehyde and osmium tetroxide, and after asphyxiation followed by perfusion.

(The minimum conductivity is given during $\mathrm{OsO}_{\star}$ perfusion (Fig. I c).)

\begin{tabular}{|c|c|c|c|c|}
\hline \multirow{2}{*}{$\begin{array}{c}\text { Expt. } \\
\text { no. }\end{array}$} & \multirow{2}{*}{$\begin{array}{c}\text { Perfusion } \\
\text { immediately after } \\
\text { circulatory arrest }\end{array}$} & \multirow{2}{*}{$\begin{array}{c}\text { Expt. } \\
\text { no. }\end{array}$} & \multicolumn{2}{|c|}{ Asphyxiation followed by perfusion } \\
\hline & & & After asphyxiation & After perfusion \\
\hline \multicolumn{5}{|c|}{ Glutaraldehyde perfusion } \\
\hline $\mathbf{I}$ & $5^{8}$ & 7 & 42 & 43 \\
\hline 2 & 61 & 8 & 48 & 57 \\
\hline 3 & 62 & 9 & 59 & 67 \\
\hline 4 & 55 & I0 & 56 & $7 \mathrm{I}$ \\
\hline 5 & 60 & I I & 49 & $5 \mathrm{I}$ \\
\hline 6 & 56 & 12 & 55 & $6 I$ \\
\hline mean & 59 & & $5 \mathrm{I}$ & $5^{8}$ \\
\hline \multicolumn{5}{|c|}{ Osmium tetroxide perfusion } \\
\hline 13 & 77 & 15 & 45 & 83 \\
\hline 14 & 54 & I 6 & 42 & 66 \\
\hline
\end{tabular}

In other experiments the preparation was first asphyxiated by opening the thorax and incising the right heart. Eight minutes later perfusion with glutaraldehyde was started (Fig. I B). Asphyxiation causes a drop in conductivity consisting of a slow early decrease followed after about I min by a much faster drop. The initial slow decline has been ascribed to the loss of blood (which has a higher conductivity than the tissue) from the vessels. The fast drop would indicate the asphyxial loss of extracellular electrolytes to the cellular elements (Van Harreveld \& Ochs, I956). After 8 min of asphyxiation the conductivity loss was somewhat larger than that after glutaraldehyde perfusion (to $48 \%$ of the oxygenated value). The tissue was then perfused with glutaraldehyde. This caused a small early increase of the conductance which may be due to the filling of the vessels with a fluid of relatively large conductivity. However, after this increase the conductivity remained relatively constant.

Table I gives information about the conductivity losses in 6 experiments during glutaraldehyde perfusion, and in 6 experiments in which the perfusion was preceded by asphyxiation. In the former experiments, which resembled the example given in Fig. I A, the conductivity dropped to a mean of $59 \%$ of the pre-perfusion value at the 
end of the perfusion period. In the second group of experiments the conductivity dropped to $51 \%$ of the original value during asphyxiation. Perfusion increased this value to a mean of $58 \%$.

Figure I C shows the conductivity changes recorded during perfusion with $\mathrm{OsO}_{4}$ in phosphate buffer. There is an initial drop in conductivity. However, after about 3 min a minimum is reached ( $54 \%$ of the pre-perfusion value) and then the conductivity increases again, reaching after Io min a value almost as high as the pre-perfusion value $(92 \%)$. This biphasic effect of $\mathrm{OsO}_{4}$ perfusion on the conductivity was also observed by Nevis \& Collins ( 1967 ). Figure I $D$ shows the effect of $\mathrm{OsO}_{4}$ perfusion after the cortex had been asphyxiated. After circulatory arrest the conductivity dropped to $49 \%$ of the original value. Perfusion causes an increase of the conductivity almost to the pre-asphyxial value $(94 \%)$. In Table I four additional experiments are summarized.

It seemed of interest to investigate the effect of $\mathrm{OsO}_{4}$ perfusion in a preparation previously perfused with glutaraldehyde since this imitates the usual sequence of fixation for electron microscopy. Figure $\mathrm{I}$ E shows the result of such an experiment. Glutaraldehyde perfusion reduced the conductivity to $54 \%$ of the original value. However, subsequent perfusion with $\mathrm{OsO}_{4}$ increased the conductivity again to $88 \%$. In a second experiment these values were 58 and $89 \%$, respectively.

\section{Electron microscopy}

It was intended to compare EMs prepared by freeze substitution published previously (Van Harreveld et al. 1965; Van Harreveld \& Malhotra, 1967; Van Harreveld \& Khattab, 1967) with micrographs of material fixed by glutaraldehyde perfusion. Since only the surface of the cerebellar and cerebral cortex could be investigated by freeze substitution, the investigation of the glutaraldehyde-fixed material has been concentrated on the molecular layer of these cortices.

Non-asphyxiated, non-exposed cerebral cortex perfused with glutaraldehyde. Figures 2-7 show examples of the molecular layer of the cerebral cortex perfused with glutaraldehyde immediately after circulatory arrest and post-fixed with $\mathrm{OsO}_{4}$. The tissue elements are well preserved. Pre-synaptic endings can be readily identified by synaptic vesicles. Electron-transparent, apparently swollen structures are present, which often can be identified as dendritic by synaptic contacts (Figs 2-4). Some of these can be recognized as dendritic trunks; many of the smaller, more or less rounded structures may be swollen dendritic spines. They run from slightly enlarged elements which have the attributes of spines (such as a spine apparatus, Fig. 7) to large electron-transparent structures which cannot be clearly identified as such. Some of the latter may be dendritic trunks cut cross-wise. In addition to these elements there are present many spines and also dendritic trunks (Fig 3, double arrow) which are not swollen. These dendritic elements are much less conspicuous than the swollen ones. The swelling may be restricted to a portion of the dendritic structure (Fig. 5). Why some dendritic elements swell and others do not is an interesting problem. A vicarious asphyxial chloride uptake either by dendrites or by fibres of Bergmann was observed in the cerebellar cortex (Van Harreveld, $196 \mathrm{r}$ ), which was explained by the assumption that a limited amount of extracellular material is taken up by those structures in which the 
asphyxial membrane changes leading to the chloride uptake occur first. A similar explanation is suggested for the present findings.

Many pre-synaptic endings have a relatively high electron density and exhibit a more or less uniform distribution of the synaptic vesicles. Frequently endings are found, however, in which the ground cytoplasm has a low electron density and the vesicles are concentrated in the centre of the structures (Fig. 3). A central position of the vesicles can be expected when the plane of sectioning runs parallel and close to the synaptic membranes. However, the relative abundance of electron-transparent cytoplasm surrounding the vesicles suggests that also in these structures swelling has occurred by the uptake of extracellular material.

The glial cells forming the pia-glial membrane and their extensions between the neural elements usually had a rather high electron density (Fig. 2). However, in occasional preparations these structures were more transparent. The glia cells were often characterized by the presence of dense granules (probably glycogen). Similar granules were also found in some elements in the neuropil (Figs 3, 4 and 7) which may be processes of these (or other) glial cells. These elements were small and not markedly electron-transparent. Also the glia surrounding the capillaries usually had a relatively high electron density and contained similar granules as found in the pia-glial membrane (Fig 7).

There are present in these micrographs numerous small profiles which are difficult to identify but are probably non-myelinated axons. They often appear in bundles (axon fields). No swelling of these elements was observed. The bodies of nervous and glial cells and large dendrites also did not show obvious swelling.

The extracellular space was restricted to the narrow slits, about ${ }_{15} \circ \AA$ wide, separating the tissue elements which have been generally described in central nervous tissue fixed or post-fixed with $\mathrm{OsO}_{4}$. This space can be most clearly demonstrated in the axon fields. Occasionally somewhat more space was present (Fig. 6).

The main difference between these EMs and those prepared by freezing the cortex within $30 \mathrm{sec}$ of circulatory arrest and substitution in $2 \% \mathrm{OsO}_{4}$ in acetone at $-85{ }^{\circ} \mathrm{C}$ (Van Harreveld \& Malhotra, 1967 ) is the presence of swollen dendrites and possibly of swollen pre-synaptic endings. The EMs of the freeze-substituted material show a more uniform electron density of the tissue elements. The extracellular space was smaller in the glutaraldehyde-perfused preparations than in non-asphyxiated freeze-substituted material and furthermore had another distribution. In the freeze-substituted material rather large irregular spaces were found in fields of non-myelinated thin axons which had a more or less rounded profile. In glutaraldehyde-fixed cortices these axons exhibited in general polygonal profiles separated from each other by rather uniform narrow slits. In freeze-substituted asphyxiated cortex a similar swelling of dendritic elements was observed as described above in glutaraldehyde-perfused cortex (Van Harreveld \& Malhotra, 1967).

Asphyxiated, non-exposed cerebral cortex perfused with glutaraldehyde. Cerebral cortical tissue perfused $8 \mathrm{~min}$ after circulatory arrest showed similar swollen elements to those found in non-asphyxiated cortex. The swelling often seemed somewhat more pronounced in the asphyxiated material (Fig 9). Many of these swollen elements 
could again be identified as dendrites by the presence of synaptic contacts. The presynaptic terminals showed in general a rather high electron density; few terminals were found which showed signs of swelling. The pia-glial membrane seemed often somewhat more electron-transparent than when observed in the non-asphyxiated material (Fig. 8). Also the glia surrounding the capillaries was often somewhat wider and less electron-dense than in the non-asphyxiated tissue (Fig. Io). The extracellular space consisted of similar narrow slits between the tissue elements to those in the nonasphyxiated tissue, which again was especially obvious in the axon fields. This is a marked difference with asphyxiated material prepared by freeze substitution, which showed a close approximation of the tissue elements, often resulting in tight junctions. The dendritic swelling in asphyxiated glutaraldehyde-perfused cortex was similar to that in asphyxiated freeze-substituted material (Van Harreveld \& Malhotra, I967).

Non-asphyxiated and asphyxiated exposed cortex perfused with glutaraldehyde. Electron micrographs of exposed cortex which had been kept for $5 \mathrm{~min}$ in a moist chamber to recover from the mechanical stimulation involved in its exposure (Van Harreveld \& Malhotra, 1967) and was either perfused immediately after circulatory arrest or after an asphyxial period, resembled those of non-exposed cortex treated in the same ways.

Non-asphyxiated and asphyxiated exposed cortex perfused with glutaraldehyde while being flooded with a salt solution. Flooding the cerebral cortex with a physiological salt solution while being asphyxiated or being invaded by spreading depression resulted in a marked swelling of dendritic elements in EMs of the molecular layer prepared by freeze substitution (Van Harreveld \& Malhotra, I967; Van Harreveld \& Khattab, I967). This swelling was not found in control cortices flooded with the salt solution but not subjected to asphyxiation or spreading depression. It was postulated that the elements which tend to take up extracellular fluid during asphyxiation and spreading depression have available under these conditions the fluid bathing the cortex, explaining the ballooning of the dendrites. It seemed of interest to fix the cortex by perfusion with glutaraldehyde while being flooded with a physiological salt solution (Ringer's solution with half the potassium ion concentration) kept at a temperature of $38^{\circ} \mathrm{C}$. Figure 15 shows an EM of the surface layer perfused immediately after circulatory arrest under these circumstances. Enormously swollen structures are present, often containing grossly swollen mitochondria and endoplasmic reticulum (Fig. 13). These structures can in many instances be identified as dendritic elements by the presence of typical contacts with pre-synaptic structures. The swelling does not involve all the dendrites. Figure $\mathrm{I} 2$ shows a swollen dendrite continuing in a spine with an appreciable electron density, synapsing with an axonal structure. Dendritic swelling may result in membrane breaks (Figs. 13, I5). The pre-synaptic endings often are relatively electron-dense but in some instances appear grossly swollen (Fig. II). The observation of such clearly swollen terminals supports the suggestion made above that pre-synaptic terminals showing an abundance of electrontransparent cytoplasm in cortex not flooded with a salt solution also have taken up extracellular material. The pia-glial membrane (Fig. I4) and the glia surrounding the capillaries participated little or not at all in the swelling. Also the small profiles of nonmyelinated axons maintained their usual electron density. 
The ballooning of the dendrites is so large that it can easily be observed with the light microscope. Figure 16 shows a photomicrograph of the exposed cortex perfused with glutaraldehyde immediately after circulatory arrest, after having been kept in a moist chamber. The molecular layer has a fine granular appearance. The photomicrograph shown in Fig. I 7 shows cortical tissue which was flooded during the perfusion. Large transparent structures are present, often containing a dark spot. The light areas represent the swollen dendritic structures, the dark spots the mitochondria often present in them. The swollen elements are found exclusively in the surface layer of the cortex, supporting the postulate that the material taken up is derived from the fluid bathing the cortex. The large vein in Fig. 17 may have denied the underlying dendrites free access to the fluid bathing the cortex, explaining the scarcity of ballooned elements at this spot. In accordance with this observation EMs of the deeper regions of the molecular layer failed to show ballooned dendrites. These micrographs resembled those of non-asphyxiated, non-flooded cortex.

Exposed and flooded cortex which was first subjected to asphyxiation for $8 \mathrm{~min}$ followed by glutaraldehyde perfusion yielded EMs which were very similar to those of cortex perfused immediately after circulatory arrest. From the observations of material flooded during asphyxiation and subjected to freeze substitution (Van Harreveld \& Malhotra, 1967) it seems likely that under these circumstances the ballooning occurs during asphyxiation and is preserved by the glutaraldehyde perfusion.

Non-asphyxiated cerebellar vermis perfused with glutaraldehyde. Figures I8-2 I show EMs of the molecular layer of the cerebellar vermis perfused with glutaraldehyde immediately after circulatory arrest. Since the tissue was cut at right angles to the direction of the gyri the numerous small profiles represent cross-sections of the granular cell axons. Figures 18 and 19 show electron-transparent elements dispersed in the neuropil. These appear to be mostly glial elements, probably processes of Bergmann fibres. Sometimes they can be shown to be direct continuations of the latter structures (Fig. I8). They do not exhibit the features of dendrites (synaptic contacts or tubules) or of synaptic endings (synaptic vesicles) and tend to envelop neural elements. The fibre of Bergmann and the end feet forming the pia-glial membrane shown in Fig. I 8 may be slightly swollen. Dendritic elements identified by synaptic contacts and neurotubules usually were not obviously swollen (Fig. 19), although occasionally grossly swollen dendrites were encountered (Fig. 21). Also the pre-synaptic endings were in general not swollen; exceptions were found, however (Fig. 20). The glia surrounding the capillaries showed little or no swelling. The glutaraldehyde-fixed nonasphyxiated material differed from that of the cerebellar tissue freeze-substituted shortly after circulatory arrest which exhibits a more uniform electron density (Van Harreveld et al. 1965 ) in the marked swelling of glial elements and in the absence of a rather abundant extracellular space in the fields of non-myelinated axons. Instead these fibres were separated by the typical slits about $150 \AA$ wide observed by many authors in glutaraldehyde-fixed material.

Asphyxiated vermis. Vermis asphyxiated for 8 min yielded EMs which were essentially similar to those of tissue perfused immediately after circulatory arrest. The fibres of Bergmann and their end feet were usually more swollen (Fig. 22). Also the glia 
surrounding the capillaries seemed often wider and less dense than in non-asphyxiated vermis. In some micrographs the glial elements in the neuropil were more swollen but in others they resembled those of non-asphyxiated tissue (Fig. 23). The extracellular spaces consisted of the same narrow slits between the tissue elements as observed in non-asphyxiated cerebellum.

The asphyxiated glutaraldehyde-fixed cerebellar material resembled the freezesubstituted tissue in the swelling of the fibres of Bergmann and their end feet and of the glia in the neuropil but differed from the latter preparations in the relatively minor involvement of the dendrites. Furthermore, asphyxiated and freeze-substituted cerebellar cortex showed a paucity of extracellular space. The elements were in general closely approximated, often resulting in tight junctions (Van Harreveld et al. 1965).

Osmium tetroxide-perfused cerebral and cerebellar cortex. Electron micrographs of cerebral and cerebellar cortex perfused immediately after circulatory arrest with osmium tetroxide were very similar to those of glutaraldehyde-perfused and $\mathrm{OsO}_{4}$ post-fixed material. The same swelling, mainly of dendrites, was observed in the cerebral cortex (Fig. 24), and of glial elements in the cerebellum. The preparations were characterized by narrow slits between the cellular elements which were particularly apparent in the axon fields. Also the electron micrographs of tissue asphyxiated before $\mathrm{OsO}_{4}$ perfusion resembled closely those of asphyxiated, glutaraldehyde-perfused and $\mathrm{OsO}_{4}$ post-fixed material.

Freeze substitution of tissue fixed by glutaraldehyde perfusion. Glutaraldehyde perfusion causes a similar drop in tissue conductivity as asphyxiation (Table r), suggesting that a loss of extracellular ions occurs. However, electron micrographs of glutaraldehydeperfused material post-fixed with $\mathrm{OsO}_{4}$ have a different appearance from those of asphyxiated tissue subjected to freeze substitution. In accordance with the conductivity changes the latter micrographs showed an almost complete loss of extracellular space. In many places, especially in axon fields, the approximation of the cellular elements was so close that tight junctions were formed, the only space left consisting of small triangular areas where three elements met (Van Harreveld et al. 1965; Van Harreveld \& Malhotra, 1967). In the glutaraldehyde-perfused and $\mathrm{OsO}_{4}$ post-fixed tissues the fibres in the axon fields are separated by about $150 \AA$ wide slits.

The possibility was considered that glutaraldehyde perfusion causes a contraction of the extracellular space similar to that produced by asphyxiation and that the slits about $\mathrm{I}_{5} \mathrm{O}-\AA$ $\AA$ wide develop during the subsequent $\mathrm{OsO}_{4}$ post-fixation, dehydration or embedding. Attempts to produce acceptable micrographs by staining glutaraldehydeperfused but not $\mathrm{OsO}_{4}$ post-fixed material with lead citrate were not successful. Glutaraldehyde-perfused material was therefore rapidly frozen and then subjected to substitution in $2 \% \mathrm{OsO}_{4}$ in acetone at $-85{ }^{\circ} \mathrm{C}$ (Van Harreveld \& Crowell, 1964). This procedure has been shown to preserve the contraction of extracellular space caused by asphyxiation (Van Harreveld et al. 1965; Van Harreveld \& Malhotra, 1966).

Figure 27 shows a micrograph of the cerebral cortex treated in this way. Swelling of dendritic elements is present. In many places the cell membranes appear more closely approximated than in post-fixed material. Often the extracellular space is formed by triangular spaces where three elements meet. The space distribution is different from 
that of the glutaraldehyde-perfused and $\mathrm{OsO}_{4}$-fixed material shown in Figs. 2-7, where the tissue elements are separated by slits about I $50 \AA$ wide. This difference is especially striking in the axon fields, but the close approximation of the tissue elements is also often present between glia elements and dendritic and presynaptic structures. The synaptic slits had the usual width of 200-250 $\AA$. Figure 26 shows an area at higher magnification; at many places the membranes are in close approximation (arrows), although at others there are narrow slits between the membranes. At still higher magnification the former areas show the tight junction structure (Fig. 28). The EMs of this material resembled closely those of asphyxiated cortex prepared by freeze substitution (Van Harreveld \& Malhotra, 1967). Blocks of the 3 mice treated in this way showed the same features.

In another series of experiments cerebral cortex perfused with glutaraldehyde and post-fixed with $\mathrm{OsO}_{4}$ was subjected to freeze substitution. In micrographs of this material slits about $I 5 \circ \AA$ wide between the tissue elements were present (Fig. 25). The possibility has to be considered that this difference in the relationship between the plasma membranes of adjacent elements is due to a difference in staining of the glutaraldehyde-perfused and freeze-substituted material with and without $\mathrm{OsO}_{4}$ postfixation. Sjöstrand ( 1960 ) has suggested that the slits in the post-fixed material are due to the exclusive staining of the inner lamellae of the tripartite membranes, so that the rest of the membranes remaining unstained would seem to form an extracellular space. In the glutaraldehyde-perfused but not post-fixed freeze-substituted material staining of both the inner and outer lamellae of the tripartite structure then could fill up this space. Against this view is the dimension of the junction in the latter material which is of the order of $150 \AA$ against that of the post-fixed tissue, measured from the cytoplasmic sides of the black lines, which was $25^{\circ}-35^{\circ} \AA$. In Fig. 25 is a region in $\mathrm{OsO}_{4}$ post-fixed tissue where the two membranes are closely approximated (double arrows). The structure so formed has a similar dimension to the most closely approximated membranes of tissue only subjected to glutaraldehyde perfusion shown in Fig. 26. It would therefore seem that the slits observed in the post-fixed material represent an actual extracellular space. This conclusion is supported by the observation of Torack (1966) that thorotrast does not enter the extracellular space in glutaraldehyde-fixed material but does enter this space, although sparsely, in $\mathrm{OsO}_{4}$-fixed tissue which shows similar slits as in the glutaraldehyde perfused and $\mathrm{OsO}_{4}$ postfixed tissue.

These experiments suggest that glutaraldehyde perfusion causes a marked reduction of the extracellular space and that the typical ${ }_{5} 50-\AA$ wide slits develop during the post-fixation with $\mathrm{OsO}_{4}$.

\section{DISCUSSION}

The possibility mentioned in the Introduction that fixation of central nervous tissue by glutaraldehyde perfusion results in a transport of extracellular material into cellular elements is supported by the present experiments. The observations on cerebral cortex which was perfused while being flooded with a physiological salt solution 
supply the most striking evidence for such a transport. The ballooning of the dendrites attests to the ability of the cellular elements to take up material from their surroundings during glutaraldehyde fixation. The excessive swelling would be due to an unlimited amount of material available in the fluid bathing the cortex. This action of glutaraldehyde resembles strikingly the effects of oxygen deprivation (and spreading depression) on cortex flooded with a salt solution which results in a similar ballooning of dendrites in freeze-substituted material (Van Harreveld \& Malhotra, i 966; Van Harreveld \& Khattab, 1967). Certain similarities in the electron micrographs of nonflooded and non-asphyxiated tissue fixed by glutaraldehyde perfusion and tissue prepared by freeze substitution and subjected to asphyxiation (and spreading depression) can therefore be expected. Indeed EMs of cortex which was not flooded during the glutaraldehyde fixation resembled asphyxiated cortex prepared by freeze substitution. There were present in the cerebral cortex electron-transparent and apparently swollen dendritic elements which suggested that during both asphyxiation (Van Harreveld \& Malhotra, 1967) and glutaraldehyde fixation a transport of extracellular material into these structures occurs. Also in the cerebellar cortex swollen elements were found after both glutaraldehyde perfusion and asphyxiation. These were mostly of a glial nature (processes of Bergmann fibres) after perfusion, although swollen dendrites were occasionally found. There are differences, however, between glutaraldehyde-perfused and $\mathrm{OsO}_{4}$ post-fixed material and asphyxiated freezesubstituted cerebral cortex. In the former apparent swelling of pre-synaptic terminals was rather frequently seen, which had not been noticed in the latter (Van Harreveld $\&$ Malhotra, 1967). In the cerebellum fixed by perfusion glial elements were primarily swollen, whereas in freeze-substituted asphyxiated tissue also many of the dendrites were enlarged (Van Harreveld et al. I965). Furthermore, in the glutaraldehyde and $\mathrm{OsO}_{4}$-fixed material there were present the slits about ${ }_{15} \circ \AA$ wide between tissue elements (especially noticeable in the axon fields) described by numerous authors in material fixed or post-fixed with $\mathrm{OsO}_{4}$, whereas in asphyxiated freeze-substituted cerebral and cerebellar cortex the tissue elements were closely approximated, often showing the tight junction structure in the contacts.

The cerebral cortical conductivity decreased markedly during glutaraldehyde perfusion to about the same value as after asphyxiation of this tissue (Table I). The conductivity drop during asphyxiation was explained by a loss of extracellular electrolytes to the intracellular compartment (Van Harreveld \& Ochs, I956), which was supported not only by an observed water transport, both with the light (Van Harreveld, r957) and electron microscope (Van Harreveld \& Malhotra, 1967) but also by a movement of chloride ions (Van Harreveld \& Schadé, 1959) into cellular elements. It would seem likely that the conductivity decrease during glutaraldehyde perfusion is the expression of a similar movement of extracellular material into the cellular compartment, although other explanations such as a reduction of ion motility cannot be excluded. The former explanation is supported by the observation that glutaraldehyde perfusion of asphyxiated cortex causes little additional change in the tissue conductivity. Once the extracellular electrolytes are transported into the cellular compartment, the glutaraldehyde perfusion cannot exert any effect through this mechanism. This 
explanation is furthermore supported by EMs of tissue perfused immediately after circulatory arrest and then subjected to freeze substitution. This tissue showed dendritic swelling and a close approximation of the plasma membranes of the tissue elements often resulting in tight junctions. The EMs obtained in this way closely resembled those from asphyxiated and freeze-substituted tissue (Van Harreveld et al. I965; Van Harreveld \& Malhotra, 1967). These observations support the concept that the conductivity decrease during glutaraldehyde perfusion is like that during asphyxiation caused by a loss of extracellular ions to the intracellular compartment.

Perfusion of cerebral tissue with $\mathrm{OsO}_{4}$ after glutaraldehyde fixation causes a rather marked conductivity increase (Table $\mathrm{I}$ ). It could be surmised that $\mathrm{OsO}_{4}$ causes a breakdown of the high-resistance membranes enabling intracellular ions to participate in the transport of the measuring current. However, another explanation of all or part of the conductivity increase would be that $\mathrm{OsO}_{4}$ creates again an extracellular space. This possibility is supported by the EMs of tissue post-fixed with $\mathrm{OsO}_{4}$ after glutaraldehyde perfusion and then subjected to freeze substitution which showed an appreciable extracellular space in the form of slits between the tissue elements in contrast to the EMs of cortex frozen after glutaraldehyde perfusion alone. As a mechanism for such an effect, one could surmise that the application of $\mathrm{OsO}_{4}$ results in the formation of a charge on the membrane. The repulsion between equal charges on opposing membranes would then create a space between the membranes. Such a mechanism could explain the rather uniform width of the slits between the tissue elements in $\mathrm{OsO}_{4}$-treated material. This effect of $\mathrm{OsO}_{4}$ would need an aqueous surrounding since the slits are lacking in glutaraldehyde-perfused asphyxiated material subjected to freeze substitution in $2 \% \mathrm{OsO}_{4}$ in acetone at $-85^{\circ} \mathrm{C}$, where the water concentration cannot exceed $2 \%$ (Van Harreveld et al. 1965). It would seem, furthermore, that the subsequent treatment of the tissue can affect the spaces postulated to have been created by $\mathrm{OsO}_{4}$ post-fixation. In material prepared by glutaraldehyde fixation and post-fixed with $\mathrm{OsO}_{4}$, Karlsson \& Schultz (1965) and Johnston \& Roots (1967) observed an abundance of closely apposed and fused surface membranes when acetone had been used for dehydration, whereas the latter authors found slits about $100 \AA$ wide between the cellular elements in tissue dehydrated with ethanol (as was also observed in the present material). Although acetone was used in freeze substitution both EMs with closely approximated cell membranes and with slits about $150 \AA$ wide were found in the present experiments depending on whether or not the tissue had been post-fixed with $\mathrm{OsO}_{4}$.

Fixing the tissue by $\mathrm{OsO}_{4}$ perfusion initially decreased the tissue conductivity, suggesting that a similar transport of extracellular material into the intracellular compartment occurs as postulated during glutaraldehyde perfusion. Indeed EMs of this material show a similar swelling of dendritic and glial elements in cerebral and cerebellar cortex as observed after glutaraldehyde fixation. However, after a few minutes this trend is reversed and the conductivity increases again. It could be surmised that this increase in conductivity is the expression of the same process which seems to create the slits between tissue elements during post-fixation of glutaraldehydeperfused material. These two processes, both elicited by the $\mathrm{OsO}_{4}$ perfusion, then 
would explain the fluid distribution in $\mathrm{OsO}_{4}$-perfused material, the swelling of dendrites and glia, and the presence of uniform slits between tissue elements.

Although electron microscopy of material prepared by perfusion fixation has contributed immeasurably to the understanding of the structure and function of the central nervous system it cannot be relied upon to reproduce faithfully the water distribution in the living tissue. On the one hand perfusion with glutaraldehyde or $\mathrm{OsO}_{4}$ seems to cause a contraction of the extracellular space by a transport of material out of the extracellular compartment into specific neuronal and glial elements; on the other hand, $\mathrm{OsO}_{4}$ seems to create a new extracellular compartment, the slits about I $50 \AA$ wide between the tissue elements. The distribution of this new extracellular material may be quite different from that of the original material in the living tissue as is suggested by a comparison of the extracellular space in the axon fields of tissue frozen within $30 \mathrm{sec}$ of circulatory arrest and subjected to substitution fixation at low temperature (Van Harreveld et al. 1965; Van Harreveld \& Malhotra, 1967) with the corresponding extracellular space in glutaraldehyde-perfused and $\mathrm{OsO}_{4}$ post-fixed material. In the former the axons are rounded with irregular spaces between them; in the latter the axons tend to be polygonal separated by uniform $150-\AA$ wide slits. As discussed previously (Van Harreveld et al. 1965), the spaces in freeze-substituted material may also have been distorted during the substitution in acetone and subsequent embedding. Although the slits in the axon fields of $\mathrm{OsO}_{4}$ fixed or post-fixed material must be interpreted with caution, the possibility is not excluded that such spaces are present between other structures in the living tissue. The presence of narrow clefts between glia and pre- and post-synaptic structures in freeze-substituted nonasphyxiated material (Van Harreveld et al. 1965; Van Harreveld \& Malhotra, 1967) would seem to support such a view. However, in freeze-dried material treated with $\mathrm{OsO}_{4}$ vapour such contacts usually showed the tight junction structure (Van Harreveld \& Malhotra, 1966).

Bennett, Pappas, Giménez \& Nakajima (1967) discussed the difficulties in distinguishing electrotonic junctions from the tight junctions caused by asphyxiation (Van Harreveld et al. 1965; Van Harreveld \& Malhotra, I967) and fixation (Karlsson $\&$ Schultz, 1965). In view of the above finding the presence of areas where the cell membranes are closely approximated in an EM exhibiting generally the $150 \AA$-wide slits between the tissue elements may indicate a membrane differentiation in these areas in that they did not participate in the formation of the $\mathrm{OsO}_{4}$-created extracellular space. Such areas may thus be valid indications of electrotonic junctions, although more reliable criteria would be highly desirable. 6698 .

This investigation was supported by a grant from the National Science Foundation, GB

\section{REFERENCES}

Bennett, M. V. L., Pappas, G. D., Giménez, M. \& Nakajima, Y. (ig67). Physiology and ultrastructure of electrotonic junctions. IV. Medullary electromotor nuclei in gymnotid fish. f. Neurophysiol. 30, 236-300.

Freeman, J. A. \& Spurlock, B. O. (1962). A new epoxy embedment for electron microscopy. 7. Cell Biol. 13, 437-443. 
Freygang, W. H. \& Landau, W. M. (1955). Some relations between resistivity and electrical activity in the cerebral cortex of the cat. F. cell. comp. Physiol. 45, 377-393.

Johnston, P. V. \& Roots, B. I. (1967). Fixation of the central nervous system by perfusion with aldehydes and its effect on the extracellular space as seen by electron microscopy. 7. Cell Sci. 2, 377-386.

Karlsson, U. \& Schultz, R. L. (1965). Fixation of the central nervous system for electron microscopy by aldehyde perfusion. 7 . Ultrastruct. Res. 12, I60-186.

LeÃo, A. A. P. \& Ferreira, H. M. (I953). Altração da impedancia elétrica no decurso da depressão alastrante de atividade do córtex cerebral. Anais Acad. bras. Cienc. 25, 259-266.

Nevis, A. H. \& Collins, G. H. (1967). Electrical impedance and volume changes in brain during preparation for electron microscopy. Brain Res. 5, 57-85.

RANCK, J. B. (1964). Specific impedance of cerebral cortex during spreading depression, and an analysis of neuronal, neuroglial, and interstitial contributions. Expl Neurol. 9, I-I6.

Reynolds, E. S. (1963). The use of lead citrate at high pH as an electron-opaque stain in electron microscopy. F. Cell Biol. 17, 208-2 I 2.

Richardson, K. C., JARETT, L. \& Finke, E. H. (1960). Embedding in epoxy resins for ultrathin sectioning in electron microscopy. Stain Technol. 35, 313-323.

SJOSTRAND, F. S. (1960). Electron microscopy of myelin and of nerve cells and tissues. In Modern Scientific Aspects of Neurology (ed. J. N. Cummings), pp. r 88-23 r. London: Edward Arnold.

Torack, R. M. (1966). The penetration of thorotrast into brain spaces following osmium, glutaraldehyde and hydroxyadipaldehyde fixation. F. Ultrastruct. Res. 14, 590-601.

VAN HaRRBVELD, A. (1 957). Changes in volume of cortical neuronal elements during asphyxiation. Am. 7. Physiol. I91, 233-242.

VAN HARREVELD, A. (I $95^{8}$ ). Changes in diameter of apical dendrites during spreading depression. Am. F. Physiol. 192, 457-463.

Van Harreveld, A. (I96I). Asphyxial changes in the cerebellar cortex. $\mathcal{F}$. cell. comp. Physiol. 57, IOI-IIO.

Van Harreveld, A. (1966). Brain Tissue Electrolytes. New York and London: Butterworth.

Van Harreveld, A. \& Crowell, J. ( 1964 ). Electron microscopy after rapid freezing on a metal surface and substitution fixation. Anat. Rec. 149, $38 \mathrm{I}-385$.

Van Harreveld, A., Crowell, J. \& Malhotra, S. K. (1965). A study of extracellular space in central nervous tissue by freeze-substitution. F. Cell Biol. 25, i $17-\mathrm{r} 37$.

Van Harreveld, A. \& Khattab, F. I. (1967). Changes in cortical extracellular space during spreading depression investigated with the electron microscope. $\mathcal{F}$. Neurophysiol. 30, 9 I I-929.

Van HaRreveld, A. \& Malhotra, S. K. (1966). Demonstration of extracellular space by freeze drying in the cerebellar molecular layer. F. Cell Sci. 1, 223-228.

VAN HarReveld, A. \& MALhOTRA, S. K. (1967). Extracellular space in the cerebral cortex of the mouse. J. Anat. ror, 197-207.

VAN HARREVELD, A. \& OCHS, S. (1956). Cerebral impedance changes after circulatory arrest. Am. F. Physiol. 187, 180-192.

VAN Harreveld, A. \& OCHS, S. (I957). Electrical and vascular concomitants of spreading depression. Am. 7. Physiol. 189, I 59-166.

Van Harreveld, A. \& Schadk, J. P. (1959). Chloride movements in cerebral cortex after circulatory arrest and during spreading depression. F. cell. comp. Physiol. 54, 65-84.

(Received 10 November 1967) 


\section{ABBREVIATIONS ON PLATES}

\author{
a axon fields \\ $b \quad$ Bergmann fibre \\ $b m$ basement membrane \\ $d$ dendrite \\ ds dendritic spine \\ $e$ endothelium
}

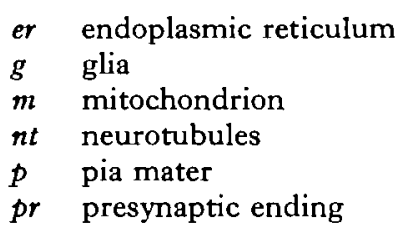

Figs. 2-6. Cerebral cortex perfused with glutaraldehyde immediately after circulatory arrest and post-fixed with $\mathrm{OsO}_{4}$. Figure 2 shows rests of the pia mater and the underlying glial membrane which is characterized by the presence of granules (probably glycogen). Elements with granules which may be glial fibres are also present in the neuropil (Figs. 2-4). Dendritic structures are identified by synapses (arrows) with axonal endings which contain synaptic vesicles. Some of the dendritic elements are transparent and appear swollen (Figs. 2-4); others, both dendritic spines and dendritic truncs (double arrow) are more electron-dense. Sometimes only part of a dendrite is swollen (Fig. 5). The neurotubules in the non-swollen part are situated close together. They fan out in the swollen section. The three presynaptic endings situated in close proximity in Fig. 3 show an electron-transparent ground cytoplasm and a central clumping of the synaptic vesicles which may indicate swelling of these structures. In other presynaptic endings the cytoplasm is more dense. Many axon fields are present in which the elements are separated by more or less uniform slits. In some instances these slits are wider and more extracellular space is present (Fig. 6). The calibration in all the micrographs is $\mathrm{I} \mu$. 
Journal of Cell Science, Vol. 3, No. 4
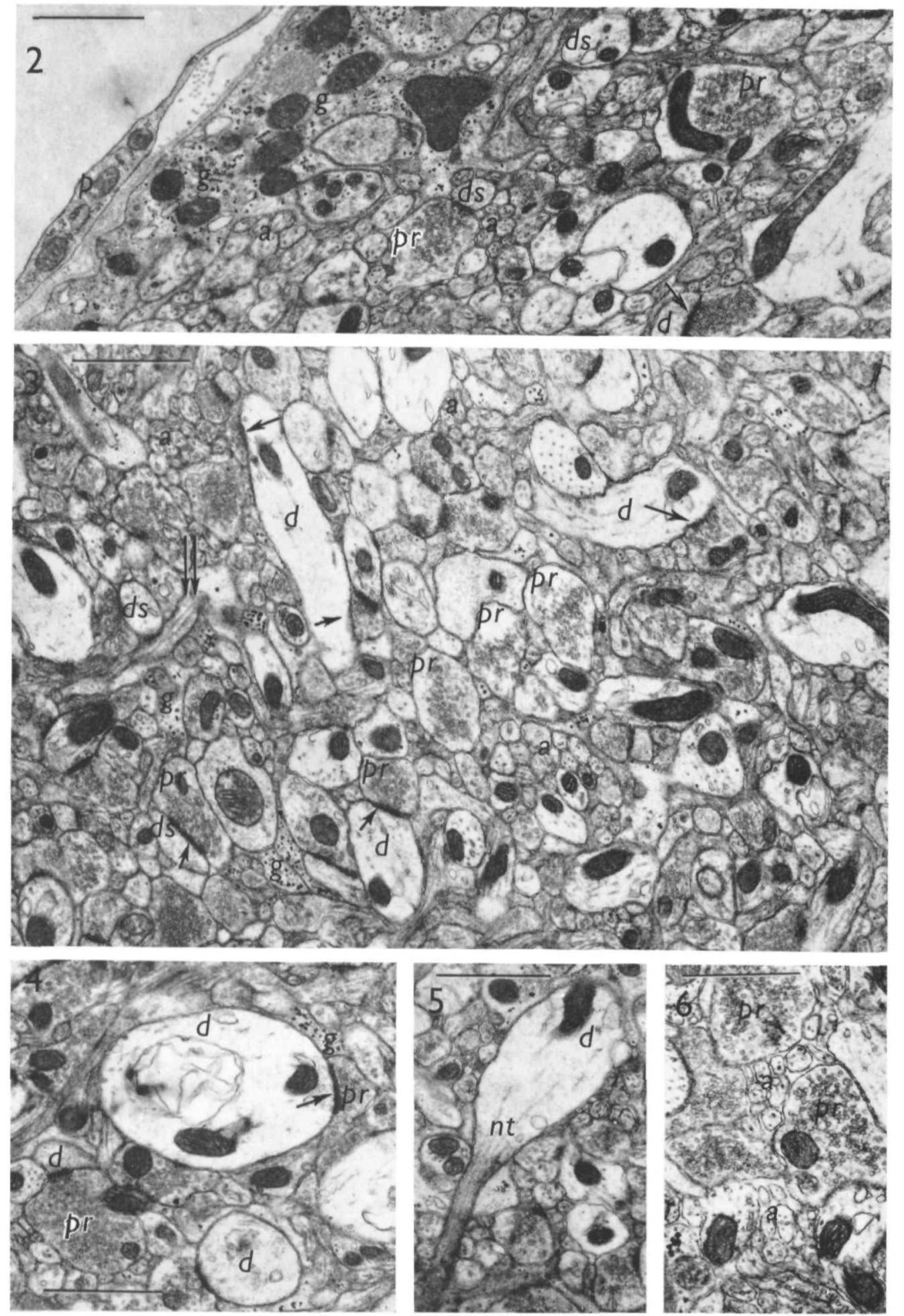

A. VAN HARREVELD AND F. I. KHATTAB

(Facing p. 594) 
Fig. 7. Cerebral cortex perfused with glutaraldehyde shortly after circulatory arrest and post-fixed with $\mathrm{OsO}_{4}$. A capillary is present showing endothelial cells and a basement membrane surrounded by glia of rather high electron density containing (glycogen) granules. An electron-transparent large element containing neurotubules is probably a dendrite. A dendritic spine contains a spine apparatus. Calibration is $\mathrm{I} \mu$.

Figs. 8,9. Cerebral cortex perfused with glutaraldehyde after $8 \mathrm{~min}$ of circulatory arrest and post-fixed with $\mathrm{OsO}_{4}$. Figure 8 shows the pia with the underlying pia-glial membrane; Fig. 9 the neuropil with swollen elements, some of which can be identified as dendrites by the synaptic connexions (arrows). The slits between the elements in the axon fields are not markedly different from those in non-asphyxiated cortex. Calibration of Figs. 8 and 9 is $1 \mu$. 
Gournal of Cell Science, Vol. 3, No. 4
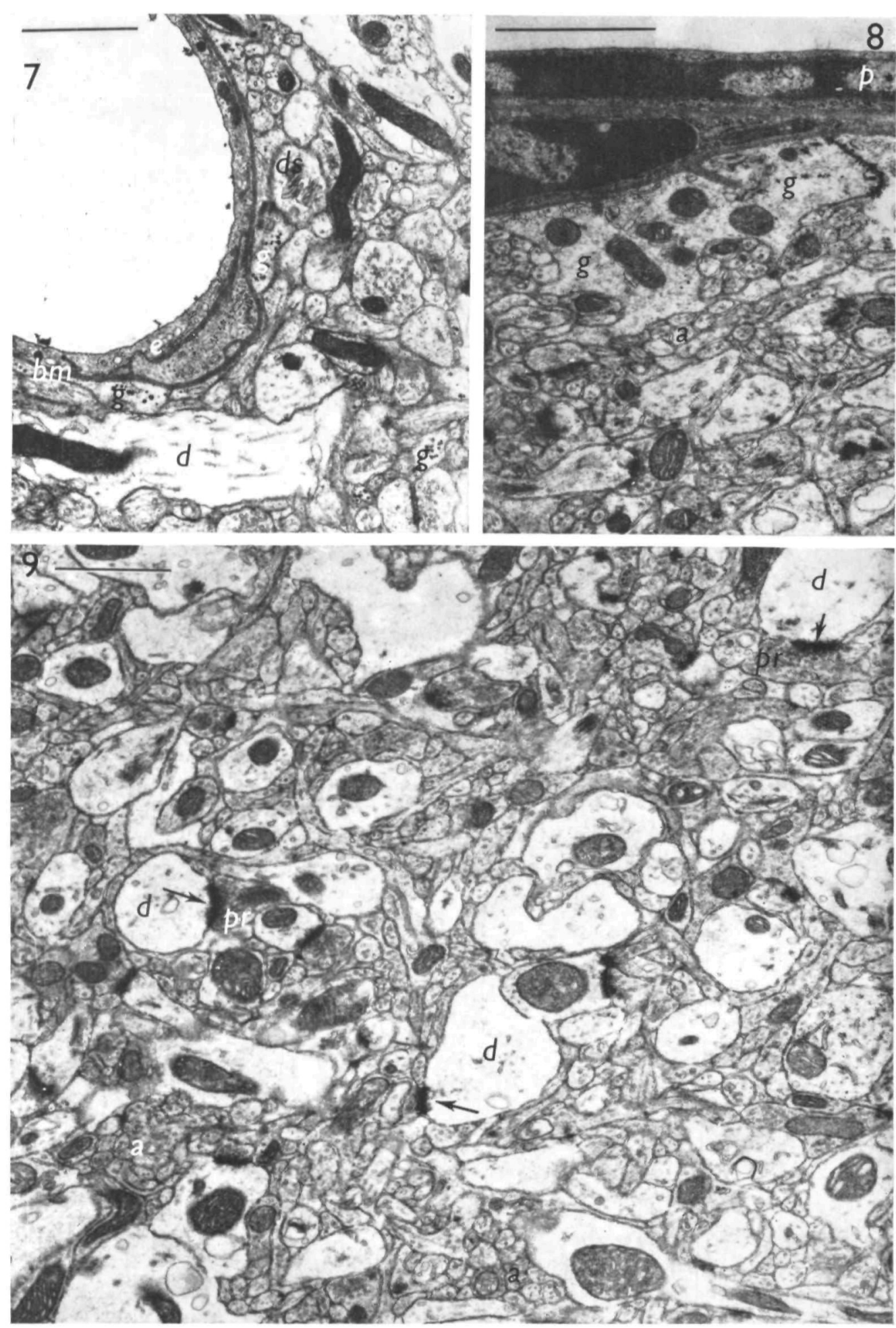

A. VAN HARREVELD AND F. I. KHATTAB 
Fig. Io. Cerebral cortex perfused with glutaraldehyde after 8 min asphyxiation $\left(\mathrm{OsO}_{4}\right.$ post-fixed). The micrograph shows a capillary surrounded by glia which contains granules (glycogen) and has a rather low electron density. This glia is probably somewhat swollen. There is a swollen dendrite identified by a synapse (arrows) visible in this section, containing a swollen mitochondrion. The slits between the small elements (axons) are similar to those of non-asphyxiated material. Calibration is $\mathrm{I} \mu$.

Figs. I I-I4. Cerebral cortex perfused with glutaraldehyde immediately after circulatory arrest while being flooded with a salt solution (post-fixed with $\mathrm{OsO}_{4}$ ). Figure I I shows a swollen presynaptic ending synapsing with a swollen dendritic element. A swollen dendrite continuing in a dendritic spine which has a high electron density and synapses with an axonal terminal is shown in Fig. 12. Figure I 3 shows ballooned dendrites, swollen endoplasmic reticulum, and broken membranes (arrows). A pre-synaptic ending contacts both dendrites. The pia mater and the underlying glia which is not markedly swollen are shown in Fig. I4. Only the dendrites show ballooning. Calibration of Figs. I I-I 4 is $1 \mu$. 
Journal of Cell Science, Vol. 3, No. 4

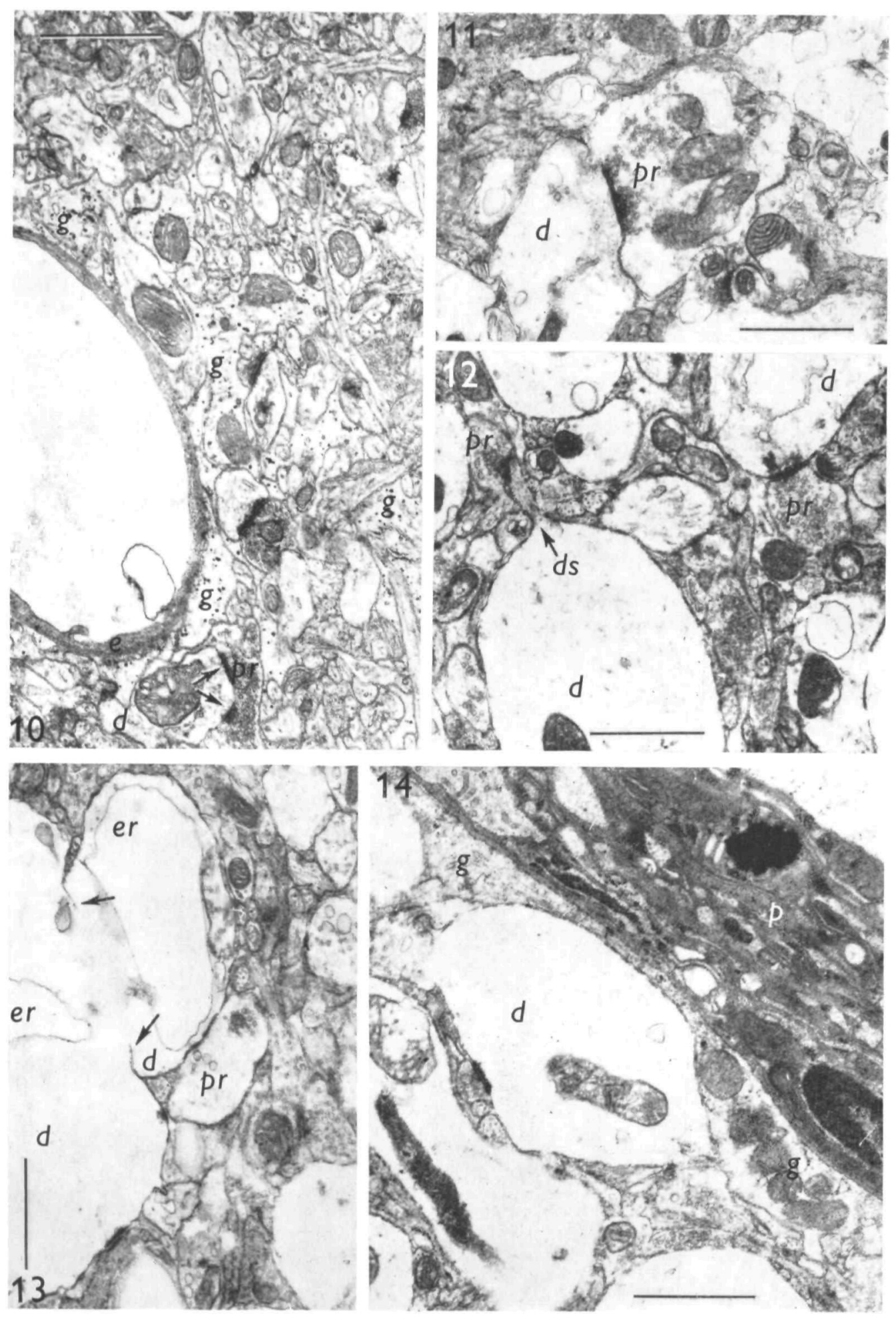

A. VAN HARREVELD AND F. I. KHATTAB 
Fig. I 5. Cerebral cortex perfused with glutaraldehyde immediately after circulatory arrest while being flooded with a salt solution (post-fixed with $\mathrm{OsO}_{4}$ ). Enormously swollen elements are present, several of which can be identified as dendritic by synapses with axonal terminals. Some of the swollen dendrites contain swollen mitochondria and endoplasmic reticulum. The membranes of the swollen structures show frequent breaks (arrows). Calibration is I $\mu$.

Figs. I6, I7. Photomicrographs of cerebral cortex perfused immediately after circulatory arrest, stained with methylene blue and azure II. The cortex yielding Fig. I 6 was not flooded with a salt solution; that yielding Fig. I 7 was flooded. The latter micrograph shows large transparent elements which correspond to the swollen dendrites in the EMs. The swelling is present only in the surface layers. A blood vessel is shown in Fig. 17. The non-flooded cortex (Fig. 16) has a granular appearance. Calibration is $5 \circ \mu$. 
Journal of Cell Science, Vol. 3 , No. 4
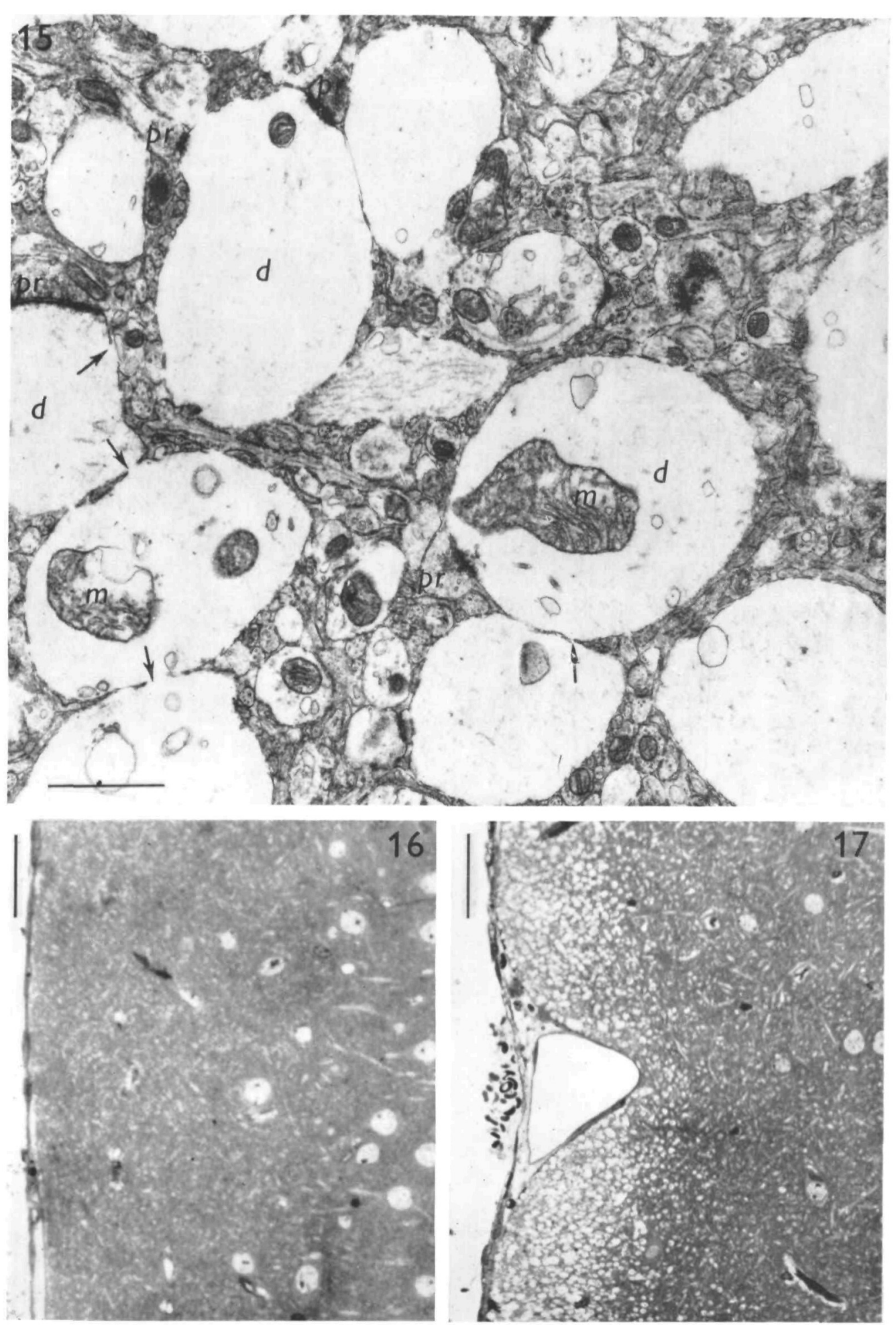

A. VAN HARREVELD AND F. I. KHATTAB 
Figs. I 8-2 I. Cerebellar vermis perfused with glutaraldehyde immediately after circulatory arrest (post-fixed with $\mathrm{OsO}_{4}$ ). Figure 18 shows the pia mater and a fibre of Bergmann forming an end foot. Processes of this structure can be followed between the neural elements. The glia elements have a relatively low electron density. The axon fields formed by granular cell axons are well developed and show the narrow slits between the elements. Figure 19 shows the neuropil deeper in the molecular layer. Glia is present which is somewhat transparent, and may represent processes of Bergmann fibres. Pre-synaptic endings can be found forming synapses with dendritic spines with a relatively high electron density. Figure 20 shows a pre-synaptic ending with transparent ground cytoplasm which probably is swollen. It synapses with a dendritic spine which has a relatively high electron density. Figure 21 shows 2 swollen dendrites as occasionally encountered. One of these can be identified by the presence of 3 synapses with axonal terminals. Calibration of Figs. I $8-20$ is I $\mu$.

Figs. 22, 23. Cerebellar vermis perfused with glutaraldehyde after $8 \mathrm{~min}$ circulatory arrest (post-fixed with $\mathrm{OsO}_{4}$ ). Figure 22 shows a swollen Bergmann fibre forming a swollen end foot under the pia. Figure 23 shows the neuropil somewhat deeper in the molecular layer. Calibration of Figs. 22 and 23 is $\mathrm{I} \mu$. 
Fournal of Cell Science, Vol. 3 , No. 4
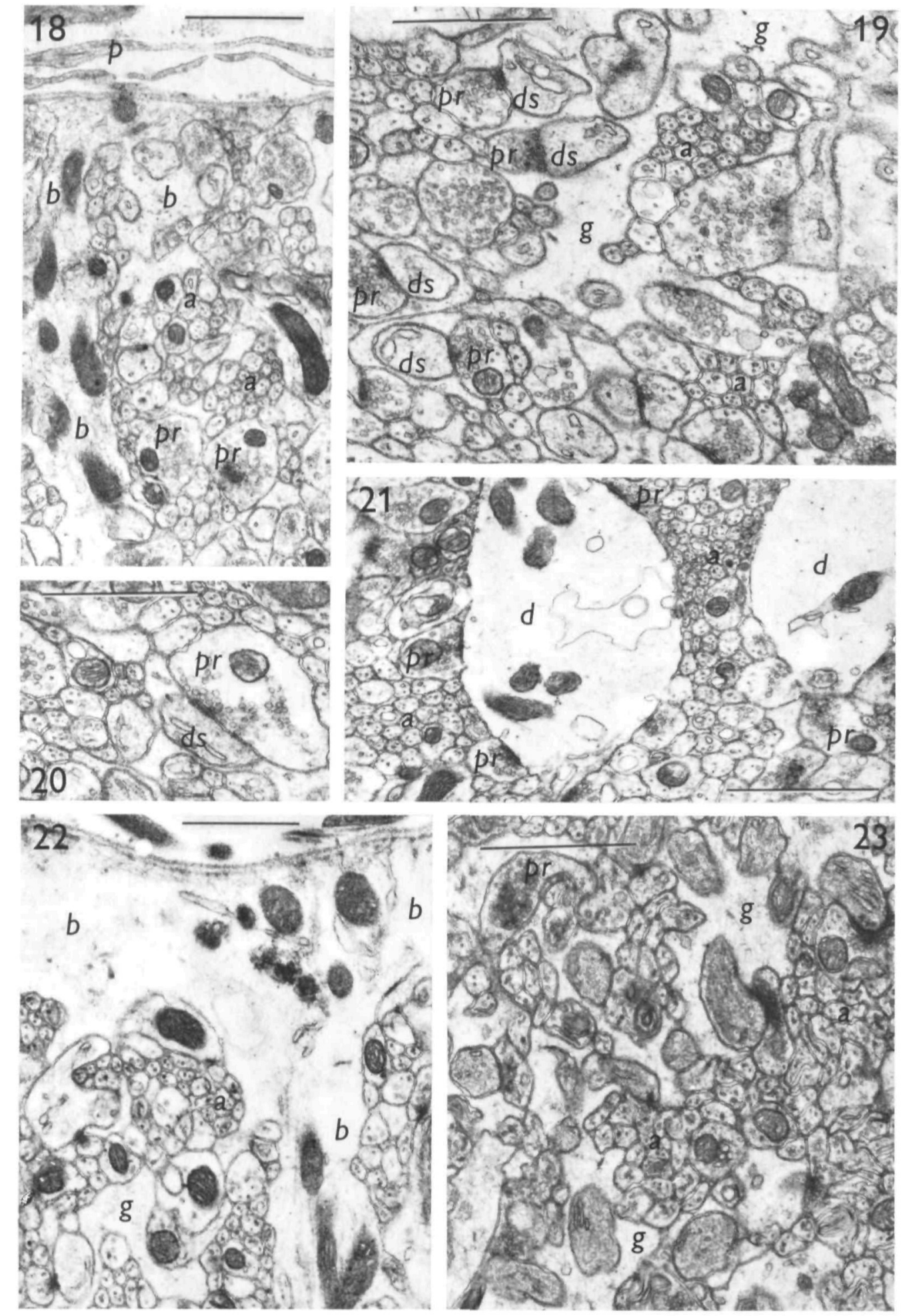

A. VAN HARREVELD AND F. I. KHATTAB 
Fig. 24. Cerebral cortex perfused with osmium tetroxide immediately after circulatory arrest. Part of the wall of a capillary is visible. There are swollen elements, one of which can be identified as a dendrite by a synapse with an axonal structure. Axon fields show rather uniform slits between the elements (calibration $I \mu$ ).

Fig. 25. Cerebral cortex, perfused with glutaraldehyde and after post-fixation with $\mathrm{OsO}_{4}$ for $2 \mathrm{~h}$, frozen and substituted in acetone at $-85^{\circ} \mathrm{C}$. An axon field is shown exhibiting uniform slits between the elements (arrows). At one spot the membranes are more closely approximated (double arrows). Calibration o I $\mu$.

Figs. 26-28. Cerebral cortex perfused with glutaraldehyde, then frozen and subjected to substitution fixation at $-85{ }^{\circ} \mathrm{C}$ in acetone with $2 \% \mathrm{OsO}_{4}$ added. Figure 27 shows swollen elements, some of which can be identified as dendrites by the presence of synapses. The typical slits between the elements in axon fields are missing in many places, the extracellular space is often reduced to triangular spaces where three elements meet (compare with Fig. 24). Figure 26 shows elements at higher magnification. At many places the cell membranes seem to have fused (arrows). Figure 28 shows the tight junction structure observed in such spots (arrows). Calibration in Fig. 27 is $1 \mu$; in Figs. 26 and 28 it is $\circ .1 \mu$. 
Journal of Cell Science, Vol. 3, No. 4

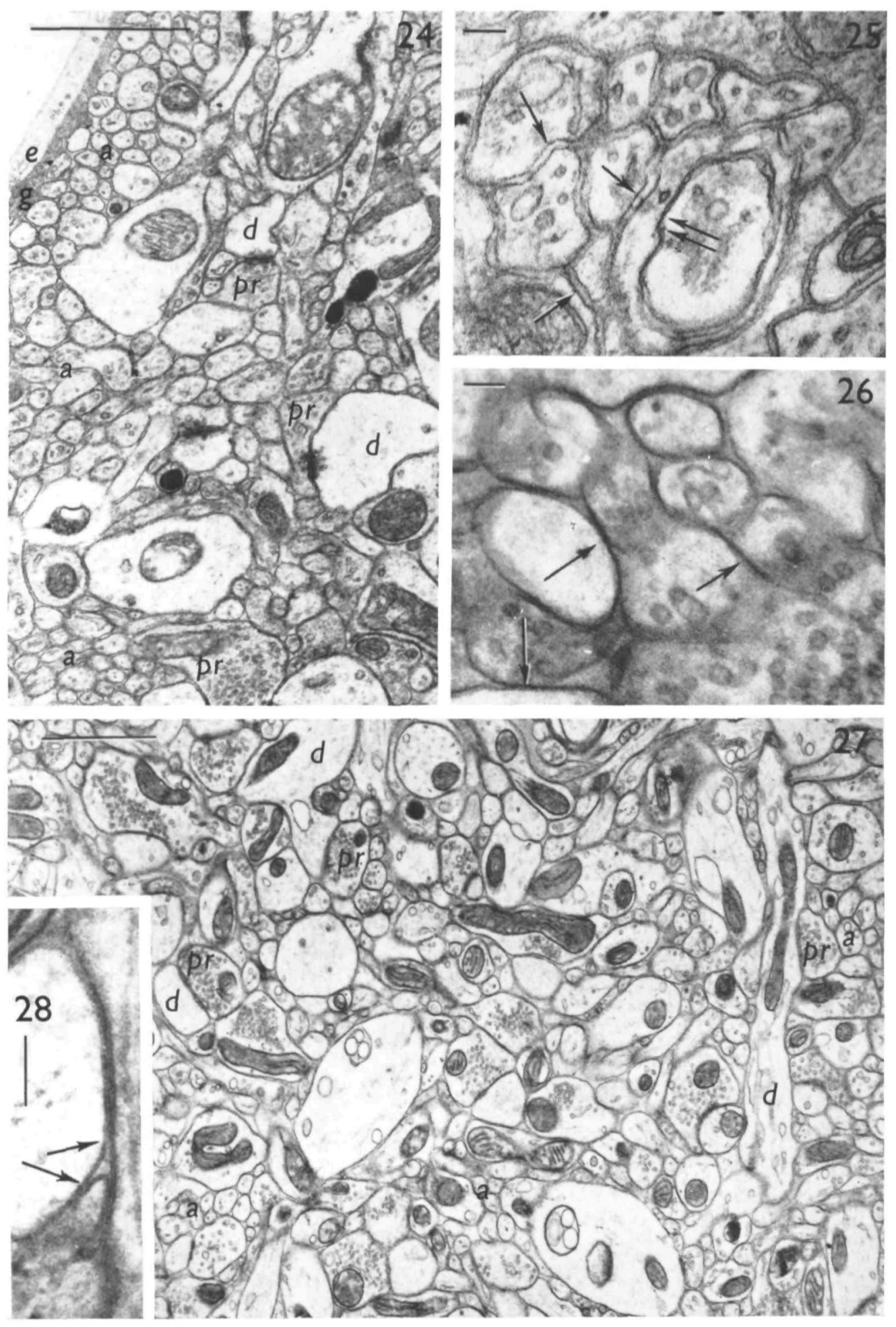

A. VAN HARREVELD AND F. I. KHATTAB 
